\title{
Dose regularization via filtering and projection: An open-source code for optimization- based proximity-effect-correction for nanoscale lithography
}

Eriksen, Emil H.; Nazir, Adnan; Balling, Peter; Vester-Petersen, Joakim; Christiansen, Rasmus E.; Sigmund, Ole; Madsen, Søren P.

Published in:

Microelectronic Engineering

Link to article, DOI:

10.1016/j.mee.2018.07.013

Publication date:

2018

Document Version

Peer reviewed version

Link back to DTU Orbit

Citation (APA):

Eriksen, E. H., Nazir, A., Balling, P., Vester-Petersen, J., Christiansen, R. E., Sigmund, O., \& Madsen, S. P. (2018). Dose regularization via filtering and projection: An open-source code for optimization-based proximityeffect-correction for nanoscale lithography. Microelectronic Engineering, 199, 52-57.

https://doi.org/10.1016/j.mee.2018.07.013

\section{General rights}

Copyright and moral rights for the publications made accessible in the public portal are retained by the authors and/or other copyright owners and it is a condition of accessing publications that users recognise and abide by the legal requirements associated with these rights.

- Users may download and print one copy of any publication from the public portal for the purpose of private study or research.

- You may not further distribute the material or use it for any profit-making activity or commercial gain

- You may freely distribute the URL identifying the publication in the public portal 
Dose regularization via filtering and projection: an open-source code for optimization-based proximity-effect-correction for nanoscale lithography

Emil H. Eriksen, ${ }^{1}$ Adnan Nazir, ${ }^{2}$ Peter Balling, ${ }^{1,2}$ Joakim Vester-Petersen, ${ }^{3}$ Rasmus E. Christiansen, ${ }^{4}$ Ole Sigmund, ${ }^{4}$ and Søren P. Madsen ${ }^{3}$

1) Department of Physics and Astronomy, Aarhus University, Ny Munkegade 120, 8000 Aarhus C, Denmark

${ }^{2)}$ Interdisciplinary Nanoscience Center, Aarhus University, Gustav Wieds Vej 14, 8000 Aarhus C, Denmark

3) Department of Engineering, Aarhus University, Inge Lehmanns Gade 10, 8000 Aarhus C, Denmark

4) Department of Mechanical Engineering, Technical University of Denmark, Nils Koppels Alle 404, 2800 Lyngby, Denmark

A new method for dose regularization in optimization-based proximity-effectcorrection is proposed. In contrast to the commonly adopted approach of adding penalty terms to the objective function, a modified scheme is demonstrated where dose regularization is achieved via filtering and projection techniques.

The resulting dose patterns are simple and two-toned, and can thus readily be applied in production. Furthermore, existing extensions developed in the context of topology optimization that build on top of the filtering framework, such as robust optimization and strict length scale control, can be adopted directly. The validity of the scheme is assessed in experiments, where the resolvable feature size of the considered $30 \mathrm{kV}$ electron-beam lithography system is decreased from around $100 \mathrm{~nm}$ to a few tens of nm. A Python implementation of the scheme is made freely available. 


\section{INTRODUCTION}

In electron-beam lithography (EBL), an electron beam is scanned over the surface of a thin layer of electron-sensitive resist. The exposure alters the physico-chemical structure of the resist, which enables selective removal of the exposed/unexposed areas in a subsequent development step (positive/negative resist). The resolution of EBL systems is limited by forward scattering of electrons as they travel through the resist along with back scattering of electrons from the substrate. While the range of the backscattered electrons is relatively long $(\approx 10 \mu \mathrm{m})$, the forward scattering is more local with a range of some tens of nanometers ${ }^{1}$. These effects, often denoted jointly as proximity effects, cause unintended exposure of regions adjacent to the intended dose pattern.

Since the 1970s, a number of proximity-effect-correction (PEC) schemes have been proposed. Dose-modification schemes, such as the self-consistent $\operatorname{method}^{2,3}$, the pattern-area density map ${ }^{1}$, and various transform ${ }^{4}$ and optimization-based ${ }^{5-7}$ approaches, modify the dose pattern based on an underlying model of the EBL process. Shape-modification schemes employ a more empirical approach where the pattern shapes are modified according to experimentally-determined design tables ${ }^{8,9}$. Other powerful techniques include background equalization methods, such as GHOST ${ }^{10}$, and various hybrid approaches ${ }^{11-13}$. While the early literature considers micrometer-sized structures, the focus of more recent research is shifting towards the nanoscale. At the nanoscale, the main contribution to the proximity effect is from forward scattering, which requires highly localized PEC. Recent studies have been addressing the sub-100-nm regime utilizing different concepts such as geometry assisted PEC design rules ${ }^{14}$ and minimal variation of critical-development time ${ }^{15}$.

In this work, we consider structures with a size below $1 \mu \mathrm{m}$ and feature sizes smaller than $100 \mathrm{~nm}$. We employ an image-based representation of the dose pattern and formulate the PEC scheme as an optimization problem. The main drawback of optimization-based approaches is that they tend to yield complex dose patterns, which are difficult to realize in practice. This issue is commonly addressed by regularization via the addition of penalty terms to the objective function ${ }^{6,7}$. In this work, an alternative dose regularization strategy, based on the filtering and projection techniques developed in the context of topology optimization $^{16,17}$, is demonstrated. The resulting dose patterns are simple and two-toned, and can thus readily be adopted in production. To demonstrate the practical applicability 
of the resulting PEC scheme, test geometries with sub-50-nm features are fabricated experimentally. In the hope of stimulating the development of open-source PEC tools, a Python implementation of the scheme is made freely available.

\section{MODELLING}

The blueprint design $B$ and the dose pattern $D$ are both two-dimensional quantities. The resulting physical structure $P$ can be approximated as a two-dimensional pattern, which is extruded perpendicular to the resist surface to form a three-dimensional object. We will use the term "physical structure" and the symbol $P$ to refer to this two-dimensional pattern. To enable a discretized formulation, applicable to numerical optimization, the resist surface is divided into $n \times m$ square elements, or pixels. The elements are arranged in a regular two-dimensional grid, like the pixels of an image. Each of the quantities $\{B, D, P\} \in Q$ can

then be expressed in discrete form as $n \times m$ element matrices $\mathbf{x}^{Q}$, where each entry $x_{i j}^{Q}$ holds the value of $Q$ evaluated at the center of pixel $(i, j)$. The blueprint design and the physical structure are both strictly binary, with 0(1) indicating the absence(presence) of material. Similarly, the dose can be considered binary with 0(1) indicating that the e-beam is off(on). However, some EBL systems support multiple dose steps, in which case the dose can take the discrete values $\{0,1 /(l-1), \ldots, 1\}$, where $l$ is the number of dose steps.

\section{A. Dose optimization}

The relation between the dose pattern $\mathbf{x}^{D}$ and the resulting physical structure $\mathbf{x}^{P}$ can formally be expressed as

$$
\mathbf{x}^{P}=\hat{T}\left(\mathbf{x}^{D}\right)
$$

where the operator $\hat{T}$ represents the forward model, i.e. a known physical model of the EBL process. The forward model used in this paper is presented in section IIC. Defining the difference between the desired blueprint design $\mathbf{x}^{B}$ and physical structure as the error, the PEC scheme can be formulated as a least-squares optimization problem,

$$
\begin{array}{ll}
\underset{\mathbf{x}^{D}}{\operatorname{minimize}} & f\left(\mathbf{x}^{D}\right)=\frac{1}{n m}\left|\mathbf{x}^{B}-\hat{T}\left(\mathbf{x}^{D}\right)\right|^{2}, \\
\text { s.t. } & x_{i j}^{D} \in\{0,1 /(l-1), \ldots, 1\} .
\end{array}
$$


Due to the discrete nature of $\mathbf{x}^{D}$ along with a possibly non-differentiable $\hat{T}$ operator, the problem in Eq. 2 is inherently non-smooth. However, the problem can be cast into a smooth form by relaxing the constraints on $x_{i j}^{D}$ to the continuos interval $[0 ; 1]$ while replacing any non-differentiable operations in $\hat{T}$ with smooth alternatives. A key advantage of this approach is that it enables the use of highly efficient gradient-based optimization techniques. As the problem size can be large, e.g. 1 mio. degrees of freedom for a $1 \times 1 \mu \mathrm{m}^{2}$ domain resolved by $1 \times 1 \mathrm{~nm}^{2}$ pixels, the efficiency of the optimization is essential. The main drawback is the added problem of mapping the values of $\mathrm{x}^{D}$ from the continous interval $[0 ; 1]$ to the discrete values $\{0,1 /(l-1), \ldots, 1\}$. The continuation scheme facilitating this process is outlined in section IID.

\section{B. Dose regularization}

Since the solution of the equation $\mathbf{x}^{B}=\hat{T}\left(\mathbf{x}^{D}\right)$ is not unique, i.e. the same physical structure can be obtained from different dose patterns, Eq. 2 is ill-posed. Solving it directly can result in noisy dose patterns with features on the length scale of the pixel size, which can be difficult to realize in practice. This problem, as well as the mapping of $\mathbf{x}^{D}$ to the allowed, discrete values, can be addressed through regularization. A commonly adopted approach is via the addition of penalty terms to the objective function ${ }^{6,7}$. However, in this work a different strategy is proposed. It is inspired by the regularization filtering approaches employed in topology optimization ${ }^{16,17}$.

We restate the optimization problem, Eq. 2, in terms of a new virtual field $\mathbf{x}^{V}$,

$$
\underset{\mathbf{x}^{V}}{\operatorname{minimize}} f\left(\mathbf{x}^{V}\right)=\frac{1}{n m}\left|\mathbf{x}^{B}-\hat{T}\left(\mathbf{x}^{D}\left(\mathbf{x}^{V}\right)\right)\right|^{2} .
$$

The virtual field has no physical meaning, but merely serves as a proxy for expressing the dose. The dose is calculated from $\mathbf{x}^{V}$ by convolution with a kernel $K$ and projection via element-wise composition with a Heaviside step function,

$$
\mathbf{x}^{D}=H_{\eta_{0}}\left(\mathbf{x}^{V} * K\right)
$$

where $\eta_{0}$ denotes the threshold value. The projection ensures that a binary dose pattern is obtained in the end. If the EBL system supports multiple dose levels, an $l$-step dose pattern can be achieved simply by replacing the Heaviside step function in Eq. 4 by its 
$l$-step equivalent. In this work, we will limit our attention to binary dose pattern, i.e. $l=$ 2. The kernel $K$ is taken to be a Gaussian of width $\sigma_{0}$.

The combination of $\sigma_{0}$ and $\eta_{0}$ provides a handle to control the complexity of the dose pattern independently of the pixel size, making it possible to wash out discretization-dependent noise. Additionally, the reduced mesh dependence smooths the landscape of the objective function, increasing both convergence speed and stability of the optimization. However, if $\sigma_{0}$ is chosen too large, small features will be rounded. For $\eta_{0}=0 / 1$, a strict enforcement of length scale is possible for the solid/void phase ${ }^{17}$. Simultaneous control of the length scale of both phases can be achieved by imposing geometrical constraints ${ }^{18}$ or by further projections $^{19}$.

\section{The EBL process}

To facilitate a clear presentation of the examples, a simple model of the EBL process is employed. However, it must be emphasized that the prosed framework can straight forwardly be applied to problems with more complex forward models. We assume that the resist is thin, so that the dependence of the exposure $E$ on the distance to the substrate can be neglected ${ }^{20}$. It can then be modeled in two-dimensional space as the convolution of the dose aimed at the resist surface with a point spread function PSF that describes the electron-scattering effects,

$$
\mathbf{x}^{E}=\mathbf{x}^{D} * \mathrm{PSF}
$$

When both forward and backward scattering are considered, a double-Gaussian is typically employed $^{10}$, but as our main focus is forward scattering, the PSF is, without loss of generality, chosen as a single Gaussian in the remainder of this work. As customary in the PEC literature, the variance $\sigma^{2}$ of the Gaussian distribution is expressed via a beam-broadening parameter $\alpha=\sqrt{2} \sigma$.

Following exposure, the resist is developed, at which point areas with exposure below a threshold value $\eta$ are removed (a positive resist is assumed, but the PEC scheme could be formulated for a negative resist as well). This step is modeled by composition with another Heaviside step function thus yielding the predicted physical material distribution,

$$
x_{i j}^{P}=H_{\eta}\left(x_{i j}^{E}\right)
$$


Hence, the forward model can be expressed as the operator $\hat{T}$,

$$
\mathbf{x}^{P}=\hat{T}\left(\mathbf{x}^{D}\right)=H_{\eta}\left(\mathbf{x}^{D} * \mathrm{PSF}\right)
$$

where $H_{\eta}$ applied element-wise.

\section{Implementation}

The PEC scheme was implemented in Python. The convolution operations were carried out as multiplications in Fourier space with the FFT operations performed by the FFTW library ${ }^{21}$. The resulting complexity of the forward model is $\mathcal{O}(n m \log (n m))$ due to the involved FFT operations. The optimizations were carried out using the L-BFGS-B algorithm ${ }^{22}$. The gradients were evaluated via the expression

$$
\frac{\partial f\left(\mathbf{x}^{D}\right)}{\partial \mathbf{x}^{D}}=\frac{1}{n m}\left[\left.\frac{\partial H_{\eta_{0}}(\xi)}{\partial \xi}\right|_{\xi=\mathbf{x}^{V}}\left[\left.2\left(\mathbf{x}^{P}-\mathbf{x}^{B}\right) \frac{\partial H_{\eta}(\xi)}{\partial \xi}\right|_{\xi=\mathbf{x}^{E}}\right] * \operatorname{PSF}\right] * K,
$$

with $\frac{\partial H_{\eta}(\xi)}{\partial \xi}$ applied element-wise, derived using the chain rule. In this form, the complexity of evaluating the Jacobian is $\mathcal{O}(n m \log (n m))$. To enable differentiation of the (nondifferentiable) Heaviside step function, it is replaced by a smooth version ${ }^{23}$,

$$
H_{\eta}^{\beta}(\xi)=\frac{\tanh (\beta \eta)+\tanh (\beta(\xi-\eta))}{\tanh (\beta \eta)+\tanh (\beta(1-\eta))}
$$

The steepness of the projection is controlled by $\beta$. Low values of $\beta$ indicate a soft projection while $\lim _{\beta \rightarrow \infty} H_{\eta}^{\beta}(\xi)=H_{\eta}(\xi)$. To limit the non-differentiality and the restriction put on design freedom by the projection we start the optimization at a low value of $\beta$, where the problem is smooth, and the dose pattern takes values in the range [0;1]. By increasing $\beta$ continuously, using the result of the previous iteration as the initial guess for the next, the dose pattern is gradually pushed towards the discrete values $\{0,1\}^{n m}$. The resulting PEC algorithm is sketched in Algorithm 1. 


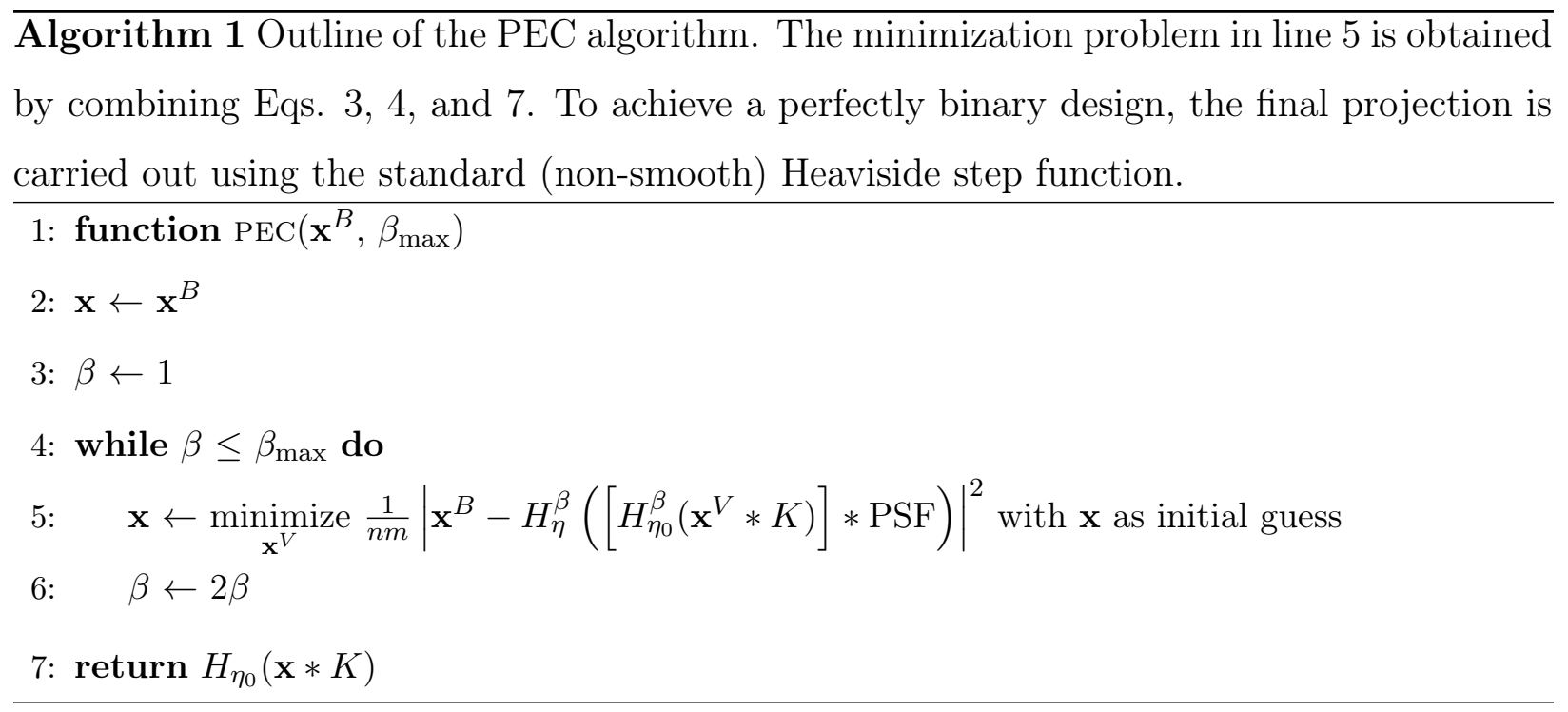

The full code is available on pypi under the package name pecpy.

\section{NUMERICAL EXAMPLES}

As the first example geometry (G1), we consider the blueprint design of Fig. 1a. It bears resemblance to the classical example of Haslam and McDonald ${ }^{4}$, but the overall size has been reduced to yield a nanoscale structure. It is represented on a $768 \mathrm{x} 768 \mathrm{~nm}^{2}$ domain,

discretized into $1 \times 1 \mathrm{~nm}^{2}$ pixels. This domain is used in all examples. The resulting PEC runtime was on the order of minutes on an Intel i7-4770 CPU.

The physical structure obtained without PEC is shown in Fig. 1e. The proximity effect is clearly visible. The large rectangles merge and their corners are rounded, while the cross is reduced to an almost rhombic shape. Applying PEC without regularization, the blueprint design is reproduced almost exactly, see Fig. 1(f). However, the dose pattern, Fig. 1(b), contains blurry features and grey-scale gradients, which are difficult to realize in practice.

Figure $1(\mathrm{c}, \mathrm{g})$ shows the effect of the proposed regularization scheme, with $\eta_{0}=0.1$ and $\sigma_{0}=10 \sqrt{2} \mathrm{~nm}$. These parameter values were found to provide a good compromise between noise reduction and feature blurring and have been used throughout this work. Compared to the case without regularization, Fig. $1(b, f)$, the error increases slightly, but it remains practically negligible. The dose pattern, on the other hand, changes drastically. The blurry features and the greyscale gradients vanish, leaving a two-toned, production-friendly dose pattern. Note that even though a particular length scale is not strictly enforced, the dose 
No PEC
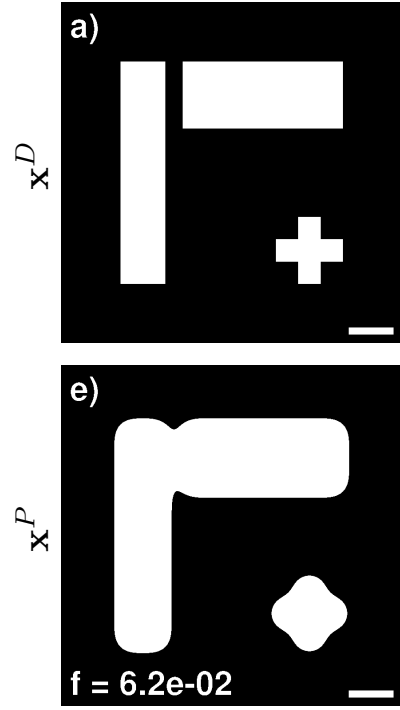

No regularization
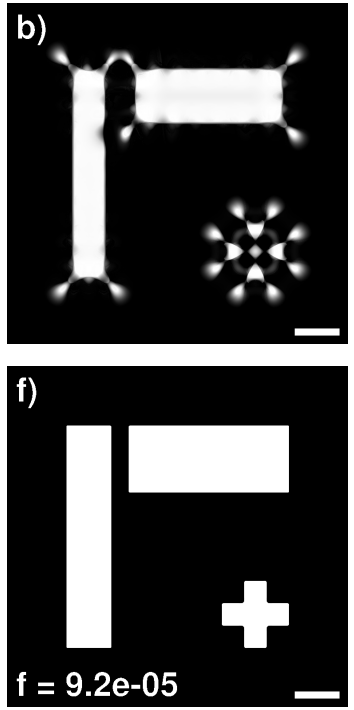

Fltr. and proj.
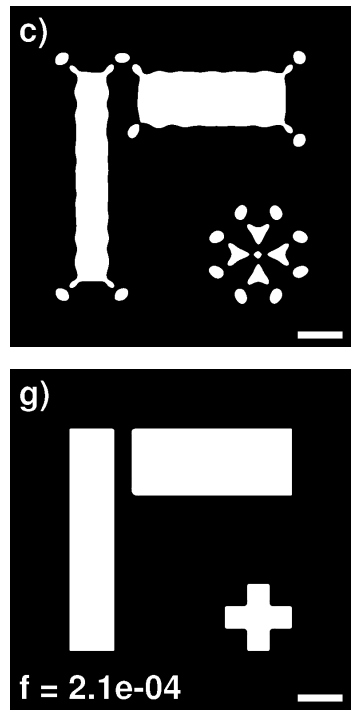

Penalty terms
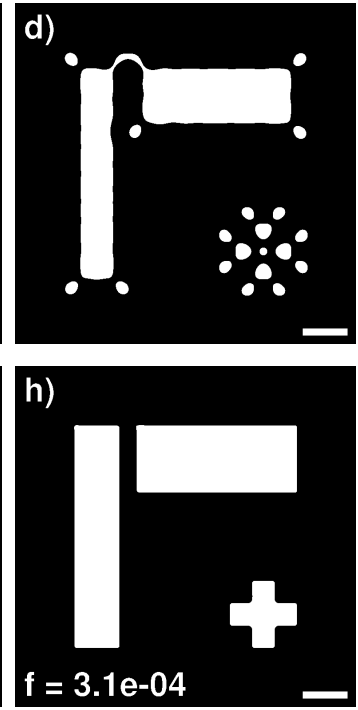

FIG. 1. Dose patterns (a-d) and resulting physical structures (e-h) with and without PEC $(\eta=$ $0.25, \alpha=30 \mathrm{~nm}$ ). The mean square error $f$ is indicated. The blueprint design is that of panel (a). The field values vary between 0 (black) and 1 (white). The scale bar is $100 \mathrm{~nm}$.

pattern appears simple and free from tiny features. These observations are qualitatively in agreement with Christiansen et al. ${ }^{24}$, where a double-filtering approach was found to yield better length scale control in practice for related topology optimization problems.

For comparison, a PEC scheme with regularization via the addition of penalty terms in the objective function was implemented. We follow the approach of Poonawala and Milanfar ${ }^{6}$ and apply a combination of a gradient term (to decrease the dose pattern complexity) and a quadratic term (to achieve a binary dose pattern). To prevent stagnation, their weight factors were turned up gradually with $\beta$. The gradient(quadratic) term improves(reduces) the smoothness of the objective function. Their individual weights must be chosen carefully to keep the optimization stabile while avoiding excessive rounding of the dose pattern, which occurs if the weight of the gradient terms becomes too large. Furthermore, as the magnitude of the gradient norm is geometry dependent, a separate calibration of the weight parameters must be carried out for each geometry to achieve optimal performance. Employing optimized weight parameters, the dose pattern in Fig 1(d,h) was obtained. Overall, the pattern complexity as well as the error is similar to Fig 1(c,g). These observations imply that the performance of the proposed regularization scheme is on par with established approaches, but with less parameter tuning needed (the same $\eta_{0^{-}}$and $\sigma_{0^{-}}$values were found 
G2
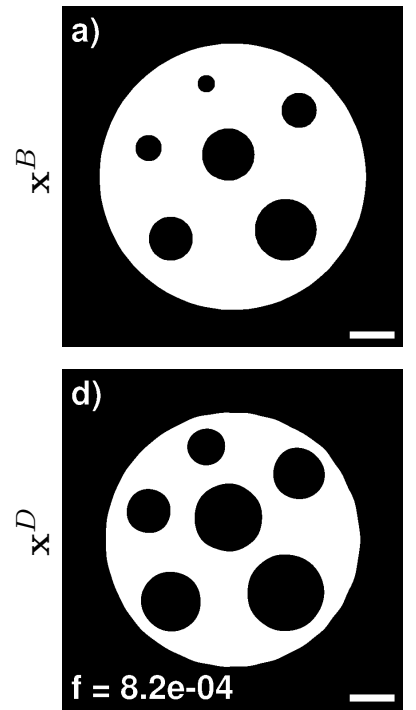

G3
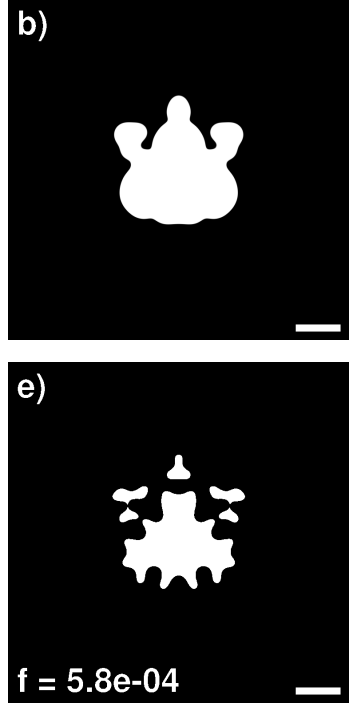

G4
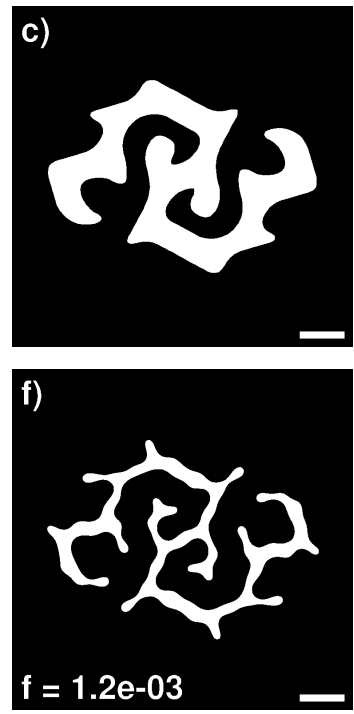

FIG. 2. Blueprint designs (a-c) and optimized dose patterns (d-f) as per Eq. 3 for different test geometries $(\eta=0.25, \alpha=30 \mathrm{~nm})$. The resulting mean square error $f$ is indicated. The field values vary between 0 (black) and 1 (white). The scale bar is $100 \mathrm{~nm}$.

be to efficient across all considered geometries). However, in contrast to the established approaches, the proposed scheme offers a suite of powerful extensions, developed in the context of topology optimization, building on top of the filtering framework. Besides strict length scale enforcement ${ }^{18,19}$ and multi-level dose projections, as mentioned in the previous sections, other relevant techniques include robust optimization ${ }^{25}$, dimensional reduction ${ }^{26}$, and unification of the device design and PEC process ${ }^{7}$.

\section{TEST GEOMETRIES}

In addition to G1, three other test geometries were selected to assess the performance of the proposed PEC scheme. The second geometry (G2) is a punctured disk with circular voids of varying size, Fig. 2(a), making it possible to estimate the minimum resolvable hole size. The third and fourth geometries (G3, G4) go beyond circular and rectangular shapes and include features in the sub-50-nm range, see Fig. 2(b,c). G3 was derived from an inhouse topologically optimized plasmonic antenna design, while G4 was inspired by Ginzburg et $a l .{ }^{27}$. G3 and G4 enable an assessment of the applicability of the PEC scheme to more exotic geometries. 
For G1, the optimized dose pattern, Fig. 1(c), is characterized by two distinct features. First, to avoid the large rectangles merging, their ends are shrunk close to the narrow gap. Similarly, dose is removed near the center of the small cross to avoid leg merging. Second, to increase the sharpness of the corners, spots of dose are added near the corners. Inspecting previous iterations of the optimization (not shown), it was found that pointy features similar to the corner-assist features proposed by Ocola et al. ${ }^{14}$ develop from the corners. In the final stages of the optimization, these features separate from the parent geometry and turn into the observed spots.

The optimized dose pattern for G2, Fig. 2(d), turns out to be rather intuitive. To avoid making the enclosing disk too large, the over-all size is reduced. Meanwhile, the size of the holes is increased to prevent overexposure. For G3 and G4, the optimized dose patterns, Fig 2(e,f), are much less intuitive. While similar strategic elements are apparent (size-reduction, fracturing), the resulting dose patterns are rather complex. They are unlike anything one would expect from manual refinement or rule-based corrections. In both cases, the blueprint design is reproduced within \pm 2 pixels of its perimeter.

\section{EXPERIMENT}

The test geometries were fabricated in gold on a silicon substrate. The substrate was spin coated with positive resist (950PMMA) and post baked at $180{ }^{\circ} \mathrm{C}$ for 5 minutes, yielding a $100 \mathrm{~nm}$ thin film. The exposure was carried out using a FEI Magellan 400 SEM system (30 kV accelerating voltage, $13 \mathrm{pA}$ current) equipped with a Raith pattern generator (100 x $100 \mu^{2}$ writing field, $4 \mathrm{~nm}$ step size). Post exposure, the samples were developed for 30 seconds in a solution (1:3) of methyl isobutyle ketone (MIBK) and isopropyl alcohol (IPA), whereas pure IPA was used as a stopper for 30 seconds. Subsequently, $2 \mathrm{~nm}$ of titanium (adhesion layer) and $20 \mathrm{~nm}$ of gold were deposited through the mask using a physical vapor

deposition system equipped with an e-beam-gun source (depositon rate $0.3 \AA / \mathrm{s}$ ). Finally, the samples were soaked in acetone to remove unwanted material. 


\section{A. Parameter fitting}

To fit the model parameters, a two-dimensional array of structures, corrected with $\eta$ and $\alpha$-values varying along each axis, was fabricated. Inspecting the resulting structures in SEM, the most accurate representation of the blueprint design was identified, and the best parameter choice was determined from its row/column number, see Fig. 3.

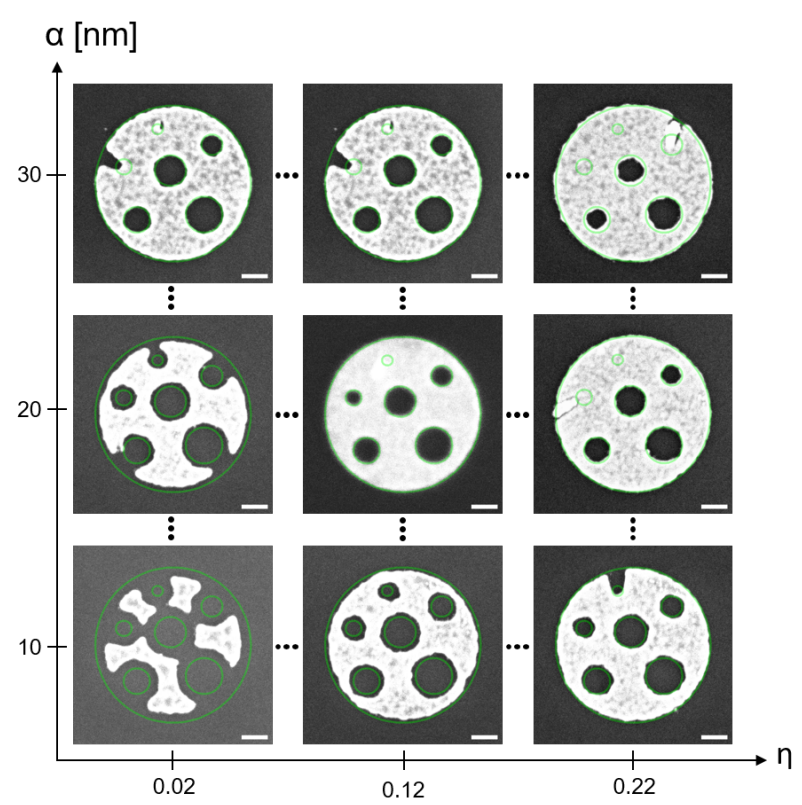

FIG. 3. Schematic illustration of a fabricated array of structures corrected using different values of $\eta$ and $\alpha$. To guide the eye, a semi-transparent outline of the intended blueprint design, Fig. 2a, is shown in green. Clearly, the best match is the center panel, i.e. the best parameter choice is $(\eta, \alpha)$ $=(0.12,20 \mathrm{~nm})$. The scale bar in each panel is $100 \mathrm{~nm}$.

In general, the model parameters can depend on all aspects of the EBL setup (substrate material, resist type and thickness, acceleration voltage and current of the electron beam, the development process, etc.). However, they do not depend on the blueprint design, so once the parameters have been determined for a particular EBL setup, any structure can be corrected. The indepence on the blueprint design was confirmed in experiment with the best parameter choice $(\eta=0.15 \pm 0.03$ and $\alpha=20 \pm 2 \mathrm{~nm})$ being equal across all geometries within measurement uncertainties. 

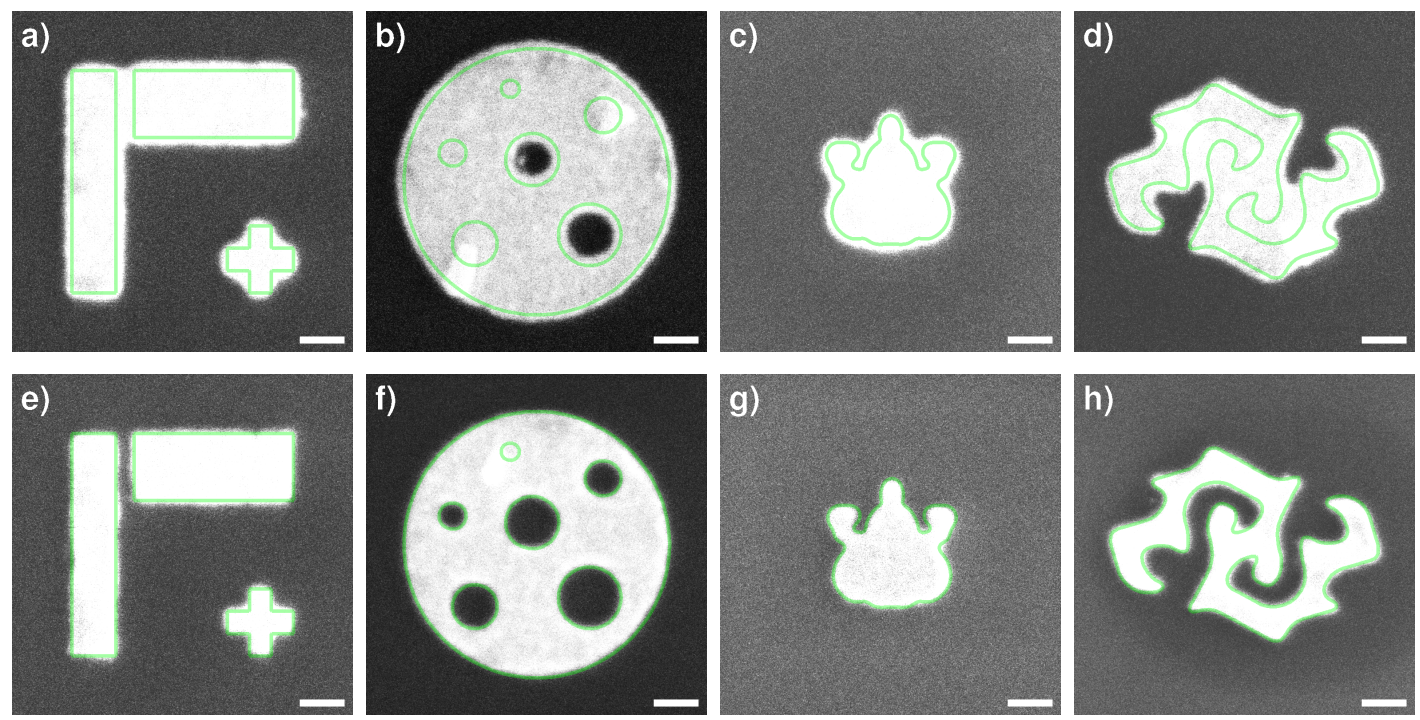

FIG. 4. (Color online) SEM images of the fabricated test structures without (a-d) and with PEC (e-h). In the correction, fitted parameter values of $\eta=0.15 \pm 0.03$ and $\alpha=20 \pm 2 \mathrm{~nm}$ were used. To guide the eye, a semi-transparent outline of the intended blueprint design is shown in green. The scale bar in each panel is $100 \mathrm{~nm}$.

\section{B. Experimental results}

Figure 4 shows the fabricated test structures with and without PEC. For G1, the proximity effect is clearly visible with the large rectangles merging, see Fig. 4(a). Additionally, the corners are rounded, and the cross is reduced to a curvy diamond-like shape. Employing PEC, Fig. 4(e), the sharpness of the corners is increased significantly, and the large rectangles detach. The edges of the cross remain slightly rounded, but the over-all cross shape is well resolved. In the case of G2, the uncorrected dose results in a disk which is slightly too large, and with only two holes resolved, see Fig. 4(b). With PEC, Fig. 4(f), the diameter of the enclosing disk is corrected, and three additional holes are resolved. We suspect that the failure to resolve the last hole is due to problems in the lift-off process.

Going to the more complex geometries, G3 and G4, features smaller than $\approx 100 \mathrm{~nm}$ tend to merge without PEC. While the over-all contours of G4 remain visible, Fig. 4(d), G3 is reduced to an almost blob-like shape, see Fig. 4(c). Upon turning on PEC, Fig. 4(g,h), all essential features of both G3 and G4 are reproduced well. The small arms of G3 now appear separated from the main body, and even the small bump at the back has become visible. The contours of G4 appear crisp, with only slight deviations near the center. 


\section{CONCLUSION AND OUTLOOK}

We have demonstrated how a new combined filtering and projection technique can be applied for dose regularization in the context of optimization-based PEC. The resulting PEC scheme yields binary, production-friendly dose patterns. The performance of the scheme was validated in experiments considering four different test geometries. A consistent performance was observed across all structures, decreasing the minimum resolvable feature size from about $100 \mathrm{~nm}$ to a few tens of $\mathrm{nm}$.

By adopting the filtering technique for dose regularization in PEC, other methods building on top of the filtering framework can be applied directly. Particularly interesting examples are multi-level dose projections, robust optimization ${ }^{25}$, strict length scale enforcement ${ }^{18,19}$, dimensional reduction ${ }^{26}$, and unification of the device design and PEC process ${ }^{7}$.

\section{ACKNOWLEDGMENTS}

This research was supported by the Innovation Fund Denmark under the SunTune project.

\section{REFERENCES}

${ }^{1}$ F. Murai, H. Yoda, S. Okazaki, N. Saitou, and Y. Sakitani, J. Vac. Sci. Technol. B 10, 3072 (1992).

${ }^{2}$ M. Parikh, J. Vac. Sci. Technol. 15, 931 (1978).

${ }^{3}$ J. Pavkovich, J. Vac. Sci. Technol. B 4, 159 (1986).

${ }^{4}$ M. Haslam and J. McDonald, J. Vac. Sci. Technol. B 4, 168 (1986).

${ }^{5}$ M. Peckerar, S. Chang, and C. Marrian, J. Vac. Sci. Technol. B 13, 2518 (1995).

${ }^{6}$ A. Poonawala and P. Milanfar, IEEE T. Image Process. 16, 774 (2007).

${ }^{7}$ M. Zhou, B. Lazarov, and O. Sigmund, Appl. Optics 53, 2720 (2014).

${ }^{8}$ H. Sewell, J. Vac. Sci. Technol. 15, 927 (1978).

${ }^{9}$ E. Kratschmer, J. Vac. Sci. Technol. 19, 1264 (1981).

${ }^{10}$ G. Owen and P. Rissman, J. Appl. Phys. 54, 3573 (1983).

${ }^{11}$ T. R. Groves, J. Vac. Sci. Technol. B 11, 2746 (1993).

${ }^{12}$ M. Osawa, K. Takahashi, M. Sato, H. Arimoto, K. Ogino, H. Hoshino, and Y. Machida, J. Vac. Sci. Technol. B 19, 2483 (2001). 
${ }^{13}$ T. Klimpel, M. Schulz, R. Zimmermann, H.-J. Stock, and A. Zepka, J. Vac. Sci. Technol. B 29, 06F315 (2011).

${ }^{14}$ L. E. Ocola, D. J. Gosztola, D. Rosenmann, and G. Lopez, J. Vac. Sci. Technol. B 33, 06FD02 (2015).

${ }^{15}$ C. Nien, L.-C. Chang, J.-H. Ye, V.-C. Su, C.-H. Wu, and C.-H. Kuan, J. Vac. Sci. Technol. B 35, 051603 (2017).

${ }^{16}$ B. Bourdin, Int. J. Numer. Meth. Eng. 50, 2143 (2001).

${ }^{17}$ F. Wang, B. S. Lazarov, and O. Sigmund, Struct. Multidiscip. O. 43, 767 (2011).

${ }^{18}$ M. Zhou, B. S. Lazarov, F. Wang, and O. Sigmund, Comput. Method. Appl. M. 293, 266 (2015).

${ }^{19}$ J. K. Guest, Comput. Method. Appl. M. 199, 123 (2009).

${ }^{20}$ S.-Y. Lee and K. Anbumony, Microelectron. Eng. 83, 336 (2006).

${ }^{21}$ M. Frigo and S. G. Johnson, P. IEEE 93, 216 (2005), special issue on "Program Generation, Optimization, and Platform Adaptation".

${ }^{22}$ C. Zhu, R. H. Byrd, P. Lu, and J. Nocedal, ACM T. Math. Software 23, 550 (1997).

${ }^{23}$ J. K. Guest, J. H. Prévost, and T. Belytschko, Int. J. Numer. Meth. Eng. 61, 238 (2004).

${ }^{24}$ R. E. Christiansen, B. S. Lazarov, J. S. Jensen, and O. Sigmund, Struct. Multidiscip. O. 52, 737 (2015).

${ }^{25}$ O. Sigmund, Acta Mech. Sinita 25, 227 (2009).

${ }^{26}$ J. K. Guest and L. C. Smith Genut, Int. J. Numer. Meth. Eng. 81, 1019 (2010).

${ }^{27}$ P. Ginzburg, N. Berkovitch, A. Nevet, I. Shor, and M. Orenstein, Nano Lett. 11, 2329 (2011). 